\title{
Cost Analysis of Hybrid Restructuration for Distribution System to Improve Voltage and Minimize Losses
}

\author{
Firas M. F. Flaih ${ }^{1}$, Lin Xiangning ${ }^{2}$, Samir M. Dawoud ${ }^{3}$, Mohammed R. Almallah ${ }^{4}$ \\ ${ }^{1,2,3}$ School of Electrical and Electronics Engineering, Huazhong University of Science and Technology, \\ Wuhan 430074, Hubei, China \\ ${ }^{1}$ General Directorate of North Distribution Electricity, Ministry of Electricity, Iraq \\ ${ }^{4}$ General Directorate of Electricity Transmission, Northern, Ministry of Electricity, Iraq \\ ${ }^{*}$ Corresponding author, e-mail: firas_flaih@hust.edu.cn
}

\begin{abstract}
Current situation in Iraqhad led to extensive blackouts which needs an expansion in generation capacity. On the other hand the government has reduced the budget allocated for energy resource development and it seems this situation will sustain for the coming years. So the fulfilment of the load demand is the biggest challenge for the ministry of electricity, Iraq with limited budget. In this paper the authors have proposed a method to reduce the power losses and therefore improve the voltage profile for low voltage (LV) distribution system that results in reduction of blackouts. The method involves the repositioning of the distribution transformer (DTR) from the existing location andthe replacement of the overhead conductor cross section area for an existing low voltage distribution system (LVDS). This method has been applied to a 20-node low voltage radial distribution network in the general directorate of north distribution electricity (GDNDE), Iraq, where voltage profile and losses are unsatisfactory. The simulation has been performed using the Matlab environment and the results demonstrate the effectiveness of the proposed method also in terms of the economic feasibility. It is observed that thesystem average voltage profile is improved by 15\%, tail end voltage enhanced by $19.7 \%$ and losses are reduced by $78 \%$ for existing the LVDS.
\end{abstract}

Keywords: low voltage distribution system (LVDS), power losses, distribution transformer (DTR), average voltage

Copyright $(2015$ Institute of Advanced Engineering and Science. All rights reserved.

\section{Introduction}

The power outage crisis in Iraq are expected to last long because of an extraordinary growing load demand due to increased population and esspecially conversion of agricultural land to residential land. Despite of the subsidized electricity tariff, some consumers increase the financial burden on the government by not paying the utilities, and this disturbs the ministry budget further leading to crisis. The import of the high power electrical appliances more worsens the crises. Furthermore the electricity theft puts more burden on the distribution network also leading towards blackout. The best solution to this problem is the expansion in the generation capacity but the current government's policy is negating this expansion. Another solution is to reduce the power losses which will improve the voltage profile and cut down the overhead costs [1]. Therefore in current scenario the energy saving scheme is better than the energy generation.An electric power system consists of three major segments, generation, transmission and distribution [2]. The electricity distribution is the final stage in the delivery (before retail) of electricity to end users [3]. The distribution networks are typically of two types, radial or interconnected.The radial network leaves the station and passes through the network area with no normal connection to any other supply, and this is typical with long rural lines to isolated load areas [3]. The major responsibility of the distribution systemwould be proper electric power distribution and guaranteeing users' normal power consumption [4]. Operating current in distribution system is much more than that in transmission systems, and hence, larger power loss (resistive) in distribution systems as compared to transmission systems [9]. With the increased loading and exploitation of the existing power structure, the probability of occurrence of voltage collapse is significantly increasing in the distribution system [10]. In distribution system and in rural areas, normally the services try to minimize wire's cross section area and 
number of poles also they install three phase DTR of large capacities on the main road closerto MV lines which leads to use of long LV lines, that main contributing factor of line lossessubsequently voltage drop. Electrical energy losses that affect electricity utilities can be classified into two categories. They are i) Technical losses-Losses due to physical aspects and ii) Non-Technical losses-Due to unauthorized line tapping or meter bypassing [11]. Several studies [5-8] introduced HVDS concept with small capacity distribution transformers to minimize technical and non-technical power losses and improve voltage of radial distribution system. S.A. Sampath Kumar et al. [3] simulated HVDS system of Kovur SSandK. Amaresh et al. [5] introduced HVDS with small capacity distribution transformers. Md Sarwar et al. [6] presented HVDS to reduce the technical power loss in distribution systems also showed the economic viability of the method. P Ravi Babu et al. [7] discussed method for reducing the non-technical losses. K. Spandana and Varsha Reddy [8] presented restructuring of existing LVDS to HVDS in agricultural field. But unfortunately all the above mentioned research lacks the economic impacts. In this paper the authors have proposed a novel method to enhance voltage profile and minimize power losses by restructuring the LV distribution system and reposition of distribution transformer (DTR) from the existing location to another that gives minimum power losses. In this paper the authors have proposed a concept to choose the best scenario in terms of reducing financial burdens for electricity sector in the public budget.

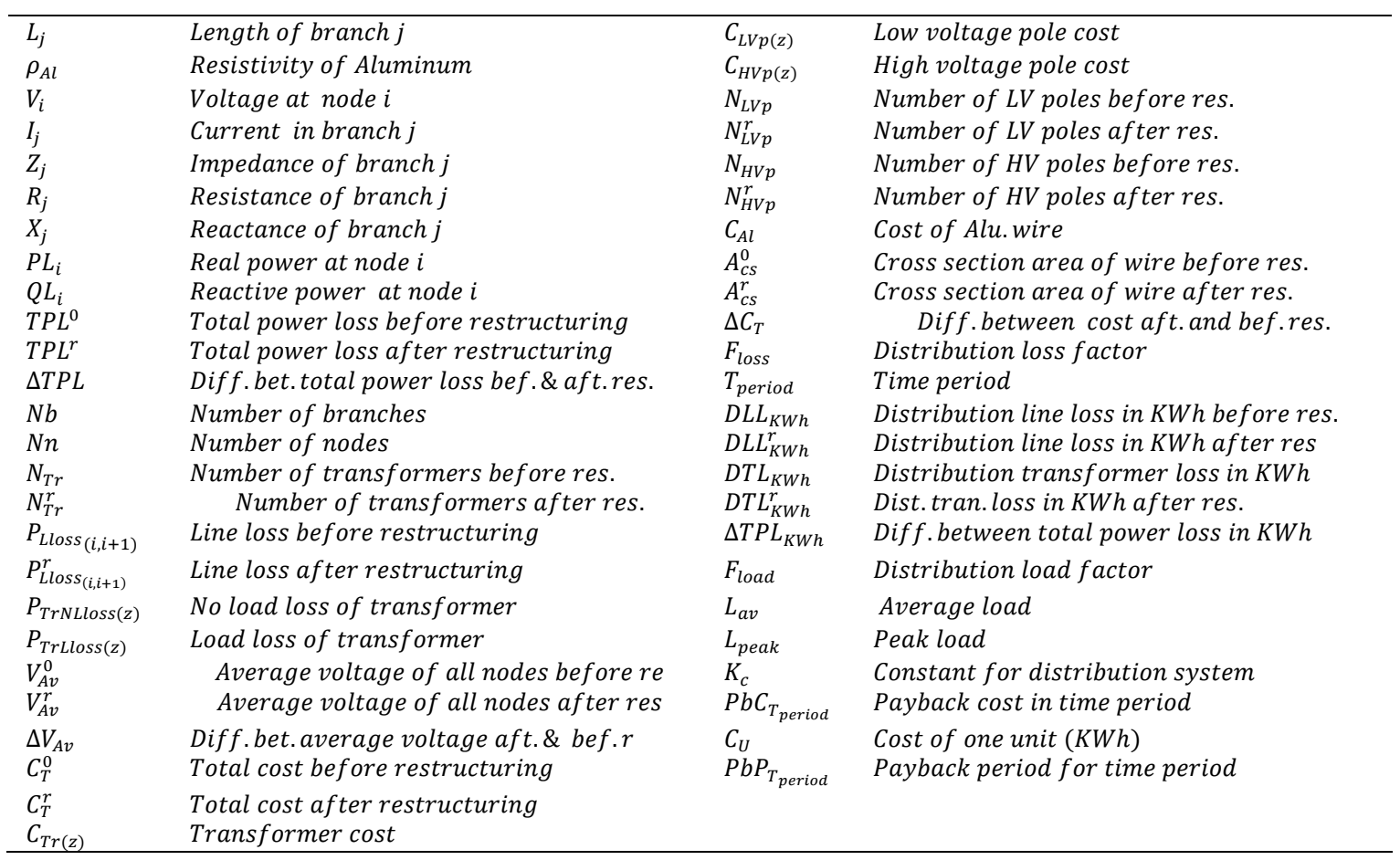

\section{Load Flow for Radial Network}

Power flow is a useful tool in operation, planning and optimization of a system. Distribution systems, generally, refers to the power system network connected to loads at lower operating voltage [12]. In this paper, the load flow calculation was done by using rectangular coordinates algorithm. It is assumed that the 3-phase radial distribution network are balanced and represented by their single line representation. Considering a 20 -node practical radial rural distribution system in GDNDE, Iraq whose single line diagram is shown in Figure 1. 


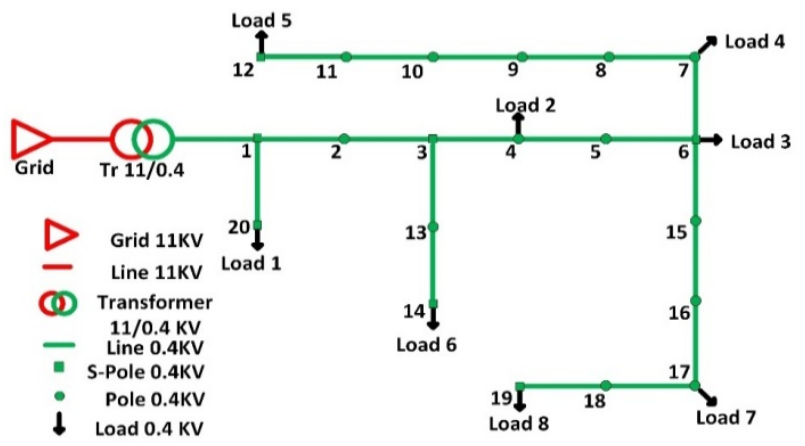

Figure 1. Single line diagram of 20 node LVDS system

\subsection{Power Loss of Radial Distribution Network}

The AC power flows are calculated by the following set of recursive equations derived from the single-line diagram in Figure 2 , the voltages at nodes $i$ and $i+1$ are $V_{i}$ and $V_{i+1}$, respectively. The current $I_{j}$ from node $i$ to node $i+1$ is given by:

$$
\begin{aligned}
& I_{j}=\frac{V_{i}-V_{i+1}}{Z_{j}} \\
& Z_{j}=R_{j}+j X_{j} \\
& P L_{i}-j Q L_{i}=V_{i+1} \cdot I_{j}
\end{aligned}
$$

From (1), (2) and (3), the voltage magnitude of $V_{i+1}$ at node $i+1$ is given by:

$$
\begin{aligned}
& V_{i+1}=\left\{\left[\left(P L_{i+1} \cdot R_{j}+Q L_{i+1} \cdot X_{j}-\frac{\left|V_{i}\right|^{2}}{2}\right)-\left(R_{j}^{2}+X_{j}^{2}\right)\left(P L_{i+1}^{2}+Q L_{i+1}^{2}\right)\right]^{\frac{1}{2}}-\left(P L_{i+1} \cdot R_{j}+\right.\right. \\
& \left.\left.Q L_{i+1} \cdot X_{j}-\frac{\left|V_{i}\right|^{2}}{2}\right)\right\}^{\frac{1}{2}}
\end{aligned}
$$

Where node $i$ has voltage $V_{i}$ and load $P L_{i}+j Q L_{i}$, the branch that is connected between node $i$ and $i+1$, is having a resistance $R_{j}$ and inductive reactance $X_{j}$. The voltages and currents should be in their permitted range.

$$
\begin{gathered}
V_{\min 1} \leq V_{\min 2} \leq V_{i} \leq V_{\max } ; i=1,2, \ldots, N n \\
0 \leq I_{j} \leq I_{\max } ; j=1,2, \ldots, N b
\end{gathered}
$$

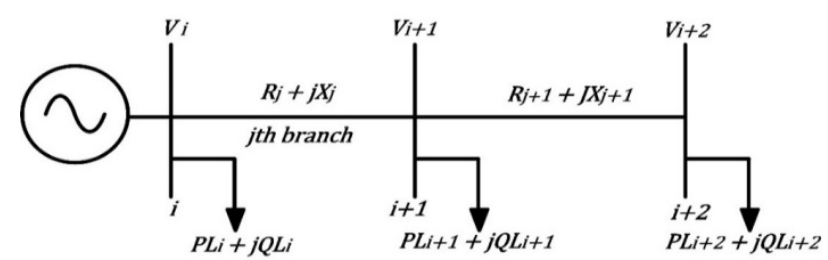

Figure 2. Simple distribution feeder

Here, $P L_{i+1}$ and $Q L_{i+1}$ represent the total real and reactive powers at the node $i+1$. The real power loss in the branch $j$ from node $i$ to node $i+1$ is given by:

$$
P_{\operatorname{Lloss}_{(i, i+1)}}=R_{j} \cdot\left(\frac{P L_{i}{ }^{2}+j Q L_{i}{ }^{2}}{\left|V_{i}\right|^{2}}\right)
$$


By summing up the losses of all branches and adding transformer load and no load loss, total real power loss before and after the restructuring can be determined as:

$$
\begin{aligned}
& T P L^{0}=\sum_{i=1}^{N b} P_{\text {Lloss }(i, i+1)}+\sum_{z=1}^{N_{T r}}\left(P_{\operatorname{TrNLloss}(z)}+P_{\operatorname{TrLloss}(z)}\right) \\
& T P L^{r}=\sum_{i=1}^{N b} P_{\text {Lloss }(i, i+1)}^{r}+\sum_{z=1}^{N_{T r}}\left(P_{\operatorname{TrNLloss}(z)}+P_{\operatorname{TrLloss}(z)}\right)
\end{aligned}
$$

By subtracting total real power loss before and after restructuring, the difference is given:

$$
\triangle T P L=T P L^{0}-T P L^{r}
$$

\subsection{Cost Analysis Calculation for RDN}

Cost analysis calculation for radial distribution network tries to find out the economic viability of the proposed method. The implementation of method requires the investment on conductors, transformers, low voltage and high voltage poles, the total cost calculation before and after restructuring is given by:

$$
\begin{aligned}
& C_{T}^{0}=\sum_{z=1}^{N_{T r} r} C_{T r(z)}+\sum_{z=1}^{N_{L V p}} C_{L V p(z)}+\sum_{z=1}^{N_{H V p}} C_{H V p(z)}+\left(K_{n} \cdot C_{A l} \cdot L_{j} \cdot A_{c S}^{0}\right) \\
& C_{T}^{r}=\sum_{z=1}^{N_{T r}^{r}} C_{T r(z)}+\sum_{z=1}^{N_{L V p}^{r}} C_{L V p(z)}+\sum_{z=1}^{N_{H V p}^{r}} C_{H V p(z)}+\left(K_{n} \cdot C_{A l} \cdot L_{j} \cdot A_{c S}^{r}\right)
\end{aligned}
$$

By subtracting total cost after and before restructuring, the difference is given:

$$
\Delta C_{T}=C_{T}^{r}-C_{T}^{0}
$$

\subsection{Average Voltage Concept}

It is difficult to deal with many node voltages to decide whether there has been a voltage improvement in distribution system or not, because some times improvement happened in some nodes and did not happened on the others, even in some cases voltage gets worse. In this paper, the authors have proposed the concept of Average Voltage to deal with all nodes in the system. The following equations are average voltage before and after restructuring:

$$
\begin{aligned}
& V_{A v}^{0}=\frac{\sum_{i=1}^{N n} V_{i}}{N n} \\
& V_{A v}^{r}=\frac{\sum_{i=1}^{N n} V_{i}^{r}}{N n} \\
& \Delta V_{A v}=V_{A v}^{r}-V_{A v}^{0}
\end{aligned}
$$

\subsection{Determination of Power Losses in KWh}

Equations of power losses in distribution lines and distribution transformers in $\mathrm{KWh}$ before and after restructure are given by:

$$
\begin{aligned}
& F_{\text {load }}=\frac{L_{a v}}{L_{\text {peak }}} \\
& F_{\text {loss }}=K_{c} \cdot F_{\text {load }}+\left(1-K_{c}\right) \cdot F_{\text {load }}^{2} \\
& D L L_{K W h}=F_{\text {loss }} \cdot \sum_{i=1}^{N b} P_{L^{\prime o s s}(i, i+1)} \cdot T_{\text {period }} \\
& D L L_{K W h}^{r}=F_{\text {loss }} \cdot \sum_{i=1}^{N b} P_{\text {Lloss }_{(i, i+1)}}^{r} \cdot T_{\text {period }} \\
& D T L_{K W h}=\left\{\left(F_{\text {loss }} \cdot \sum_{z=1}^{N_{T r}} P_{\operatorname{TrLloss}(z)}\right)+P_{\text {TrNLloss }}\right\} \cdot T_{\text {period }} \\
& D T L_{K W h}^{r}=\left\{\left(F_{\text {loss }} \cdot \sum_{z=1}^{N_{T r}} P_{T r L l o s s(z)}^{r}\right)+P_{T r N L l o s s}^{r}\right\} \cdot T_{\text {period }}
\end{aligned}
$$

TELKOMNIKA Vol. 16, No. 3, December $2015: 431-438$ 
Total power losses in $\mathrm{KWh}$ in distribution power system are the summation of distribution lines losses in $\mathrm{KWh}$ with distribution transformers losses in $\mathrm{KWh}$ is given by:

$$
\begin{aligned}
& T P L_{K W h}^{0}=D L L_{K W h}+D T L_{K W h} \\
& T P L_{K W h}^{r}=D L L_{K W h}^{r}+D T L_{K W h}^{r} \\
& \triangle T P L_{K W h}=T P L_{K W h}^{0}-T P L_{K W h}^{r}
\end{aligned}
$$

\subsection{Payback Cost \& Payback Period}

Payback cost is the difference between total power losses in kilowatt hour before and after restructuring in equations (21) and (22) multiplied by price of one unit as shown:

$$
P b C_{T_{\text {period }}}=\Delta T P L_{K W h} \cdot C_{U}
$$

Payback period is the ratio between the differences in total cost after and before restructuring in Equation (11) to payback cost, it is important to know after how many days, months or years the restructuring cover its expenses.

$$
P b P_{T_{\text {period }}}=\frac{\Delta C_{T}}{{ }^{P b C_{\text {period }}}}
$$

\section{Case Study}

A case study was made by given an existing distribution system of $416 \mathrm{~V}$ in Figure 1 . The low voltage distribution system which would run up to the customer are restructured by: (I) the length of a low voltage line are replaced by low voltage line with cross section area larger than the initial case. (II) Relocation in position of distribution transformer (11/0.416). (III) By taking best of case 1 and best of case 2. The details of the LVDS are shown in Table 1.

Table 1. Details of the LVDS

\begin{tabular}{lll}
\hline No. & \multicolumn{1}{c}{ Particulars } & \multicolumn{1}{c}{ Remarks } \\
\hline 1 & Number of transformers (11/0.416) & 1 \\
2 & Capacity of transformer & $250 \mathrm{KVA}$ \\
3 & Nature of load on transformer & under loaded \\
4 & Length of the LT lines & $950 \mathrm{~m}$ \\
5 & Number of connected loads & 8 \\
6 & Sum of connected loads & $237 \mathrm{KVA}$ \\
7 & Distance between two poles & $50 \mathrm{~m}$ \\
8 & Number of LV poles & 20 \\
9 & Number of LV branches & 19 \\
10 & Resistance of each branches & $0.028 \Omega$ \\
11 & Initial cost & $13108 \mathrm{USD}$ \\
12 & Cost of KWh & $0.025 \mathrm{USD}$ \\
13 & Aluminum wire resistivity & $2.8 \times 10^{-8}$ \\
14 & Cost of LV pole. & 416 USD \\
15 & Cost 250 KVA Tr. & 3333 USD \\
\hline
\end{tabular}

The voltage profile, the average voltage of 20 nodes, total power losses in $\mathrm{KW}$, cost in USDollar and payback period before restructuration are given in Table 2 in a column of CASE0.

\subsection{Scenario 1}

In the following LVDS, the cross section area of low voltage lines conductor is converted to value larger than the initial lines conductor size. Changing in cross section area will lead to a change in resistance of branches thus the voltage drop will decrease and enhancing voltage profile then minimizing in power losses. The conductor cross section area that give reasonable cost (Equilibrium Point) is chosen. 


\subsection{Scenario 2}

Relocation of distribution transformer into each node in the system and installation of new network considering existing LV poles and exchanging specific of LV poles with new HV poles including its extensions. The transformer relocation cost depends on the new position. DTR repositioned at every 20 nodes and position of minimum power losses is chosen.

\subsection{Scenario 3}

To give the result of case2 additional improvement in voltage profile to reach $V_{\min 2}$ and extra minimizing in power losses, hybrid case is proposed by taking the best point in case 1 and added to best location in case2.

\section{Simulation Results and Discussion}

The following results were obtained with the proposed method on LV distribution network, the voltages ranges area $0.9 \mathrm{pu}(374 \mathrm{~V}) \leq 0.95 \mathrm{pu}(395 \mathrm{~V}) \leq V_{i} \leq 1 \mathrm{pu}(416 \mathrm{~V})$, simulation results can be classified into three cases:

\subsection{Case1}

By restructuring the cross section area of conductor for each branch from 50 to 120 $\mathrm{mm}^{2}$ the conductor resistance change also from 0.028 to $0.011 \Omega$, the system average voltage is enhanced from 351.03 to 392.92 Volt, the total power losses minimized from 51.39 to 18.14 $\mathrm{KW}$. The average voltage enhanced by $12 \%$ and the loss reduced by $64.7 \%$, but the cost increased by $15.44 \%$. Therefore it is not wise to take the best voltage while the cost is very high, for this reason the authors took the voltage at equilibrium point at $70 \mathrm{~mm}^{2}$ cross section area that give $373.54 \mathrm{~V}$ average voltage, $32.28 \mathrm{KW}$ total power losses. As shown in Table 2 in a column of CASE1. Where base cost is $22333 \mathrm{USD}$, base TPL is $52 \mathrm{KW}$ and base voltage is $416 \mathrm{~V}$.

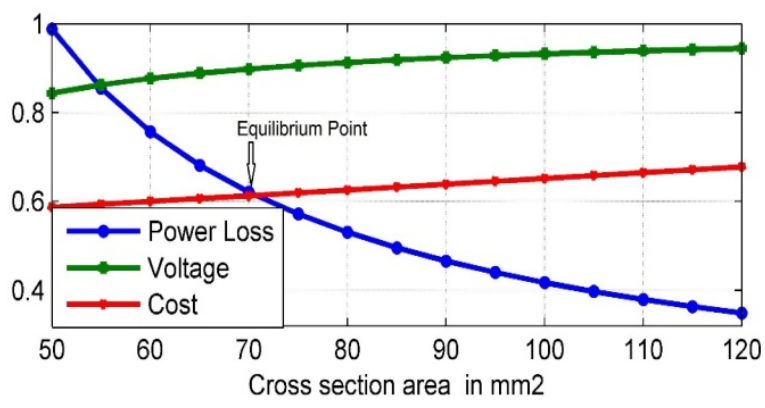

Figure 3. Cross section area of Alum. Conductor with Power Loss, Avg. Voltage and Cost

\subsection{Case2}

The 250 KVA DTR located in the main road near to medium voltage (MV) lines atnode1 as shown in Figure 1. They used long low voltage (LV) lines that leads voltage drop and power losses. In case 2 the authors tried to find at which node can relocate the DTR that give minimum power losses. The relocation of DTR leads also to restructure in some LV poles and change to HV poles, the cost was calculated for each reposition step. From simulation results, the best position that give minimum power losses is in node 5 that is $14.78 \mathrm{KW}$, the relocation of DTR to node 5 considering restructuring of $4 \mathrm{LV}$ poles to $\mathrm{HV}$ poles plus installation of $4 \mathrm{HV}$ branches, cost of that restructuringwas 14775 USD and the power losses were minimum. In this case, all node voltages is above $0.9 \mathrm{PU}$ and only voltage of six nodes is less than $0.95 \mathrm{pu}\left(V_{\min 2}\right)$ as shown in Table 2 in a column of CASE2.

\subsection{Case3}

For more enhancement in voltage profile and furtherminimization in power losses, in this case the authors took the best position in case 2 with result of case 1 at Equilibrium Point that mean repositionof the $250 \mathrm{KVA}$ DTR into node 5 considering restructuring of $4 \mathrm{LV}$ poles to HV 
poles plus installation of $4 \mathrm{HV}$ branches and also restructuring wire cross section area of LV lines. In this case the voltage of all nodes is above $0.95 \mathrm{PU}, \mathrm{TPL}$ is $11.27 \mathrm{KW}$, average voltage of all system nodes is 404 volts, and tail end voltage at node 19 improved upto $19.7 \%$. The results of 3-cases with reference case are shown in Table 2. Figure 4 showing voltage magnitude comparison of study case.

Table 2. Results of three-cases with reffrence case

\begin{tabular}{|c|c|c|c|c|}
\hline & CASE0 & CASE1 & CASE2 & CASE3 \\
\hline V1 & 416.00 & 416.00 & 401.37 & 405.66 \\
\hline V2 & 398.99 & 404.84 & 403.82 & 407.39 \\
\hline V3 & 382.04 & 393.70 & 406.27 & 409.12 \\
\hline V4 & 366.59 & 383.60 & 410.11 & 411.83 \\
\hline V5 & 353.47 & 375.08 & 416.00 & 416.00 \\
\hline V6 & 340.39 & 366.57 & 405.17 & 408.40 \\
\hline v7 & 335.24 & 363.21 & 400.90 & 405.41 \\
\hline V8 & 332.17 & 361.24 & 398.38 & 403.65 \\
\hline v9 & 329.11 & 359.26 & 395.86 & 401.89 \\
\hline V10 & 326.05 & 357.28 & 393.34 & 400.12 \\
\hline V11 & 322.99 & 355.30 & 390.82 & 398.36 \\
\hline V12 & 319.94 & 353.33 & 388.30 & 396.60 \\
\hline V13 & 380.56 & 392.68 & 404.89 & 408.14 \\
\hline V14 & 379.09 & 391.66 & 403.50 & 407.16 \\
\hline V15 & 334.69 & 362.89 & 400.48 & 405.13 \\
\hline V16 & 329.01 & 359.22 & 395.80 & 401.86 \\
\hline V17 & 323.33 & 355.55 & 391.12 & 398.59 \\
\hline V18 & 320.24 & 353.56 & 388.59 & 396.82 \\
\hline V19 & 317.16 & 351.58 & 386.06 & 395.05 \\
\hline V20 & 413.63 & 414.31 & 398.92 & 403.94 \\
\hline$V_{A v}$ & 351.03 & 373.54 & 398.98 & 404.06 \\
\hline TPLinKW & 51.39 & 32.28 & 14.78 & 11.27 \\
\hline$C_{T}$ inUSD & 13108 & 13683 & 14775 & 15350 \\
\hline$\Delta \boldsymbol{V}_{A v}$ & - & 22.51 & 47.95 & 53.03 \\
\hline$\triangle T P L$ & - & 19.11 & 36.61 & 40.12 \\
\hline$\Delta C_{T}$ in $U S D$ & - & 575 & 1666 & 2241 \\
\hline PbPinmonths & - & 5.04 & 7.50 & 9.32 \\
\hline
\end{tabular}

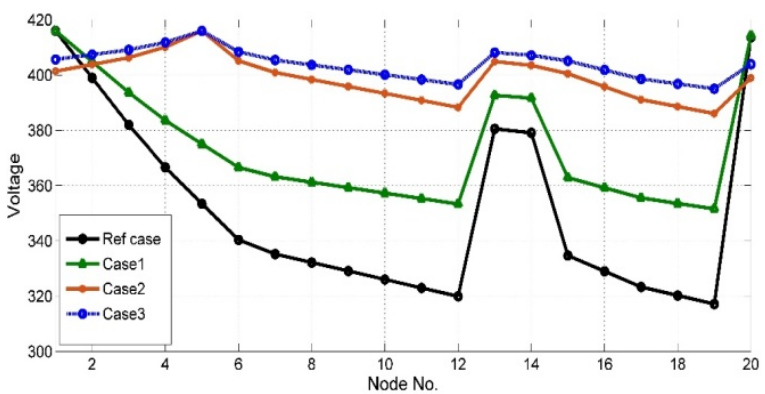

Figure 4. Voltage magnitude comparison of 20-node system

Table 3. Load Data

\begin{tabular}{|c|c|c|c|c|}
\hline Load No. & Pole No. & $P$ in $K W$ & $Q$ in KVAR & Load in KVA \\
\hline Load 1 & 20 & 29.75 & 18.43 & 35 \\
\hline Load 2 & 4 & 25.50 & 15.80 & 30 \\
\hline Load 3 & 6 & 22.95 & 14.22 & 27 \\
\hline Load 4 & 7 & 21.25 & 13.17 & 25 \\
\hline Load 5 & 12 & 29.75 & 18.43 & 35 \\
\hline Load 6 & 14 & 17.00 & 10.53 & 20 \\
\hline Load 7 & 17 & 25.50 & 15.80 & 30 \\
\hline Load 8 & 19 & 29.75 & 18.43 & 35 \\
\hline
\end{tabular}

Table 4. Load Particulars of Transformers (11/0.4) Losses

\begin{tabular}{cccc}
\hline No. & KVA rating & Tr. no load losses KW & Tr. load losses KW \\
\hline 1 & 30 & 0.10 & 0.60 \\
2 & 50 & 0.13 & 0.87
\end{tabular}




\begin{tabular}{cccc}
3 & 63 & 0.15 & 1.04 \\
4 & 80 & 0.18 & 1.25 \\
5 & 100 & 0.20 & 1.50 \\
6 & 125 & 0.24 & 1.80 \\
7 & 160 & 0.28 & 2.20 \\
8 & 200 & 0.34 & 2.60 \\
9 & 250 & 0.40 & 3.05 \\
\hline
\end{tabular}

\section{Conclusion}

Based on the case study results, voltage profile of case 3 for all nodes is more than 0.95 PU and much more efficient than the other cases. In the light of above reduced financial allocations, the proposed case 3 enhancedsystem average voltage profile by $15 \%$, reduce thepowerlosses of LVDS by $78 \%$ and improved the tail end voltage by $19.7 \%$. The payback period for current method is about 9 months only. The proposed method, enhances voltage profile and consequently enhancesthe system performance. Since losses are reduced considerably, power can be supplied to additional loads without any further expenditure in generation sector. Moreover applying this method can reduce fuel cost, which also contributes to reducing $\mathrm{CO} 2$ emissions. This method can also be applied to other distribution systems to get same benefits.

\section{Acknowledgements}

The authors gratefully thank the staff of School of Electrical \& Electronics Engineering / Huazhong University of Science \& Technology and people who assisted in this work. Special thanks to General Directorate of North Distribution Electricity /Ministry of Electricity / Iraq for their support.

\section{References}

[1] Djossou Adeyemi Amon. A Modified Bat Algorithm for Power Loss Reduction in Electrical Distribution System. TELKOMNIKA Indonesian Journal of Electrical Engineering. 2015; 14(1): 55-61.

[2] Dr P Ravi Babu, Sushma B. Operation and Control of Electrical Distribution System with Extra Voltage to minimize the Losses. Power, Energy and Control (ICPEC). 2013: 165-169.

[3] SA Sampath Kumar, V Vasudaven, J Antony, Madhu Sudhana Raju, L Ramesh. Minimization of Power Losses in Distribution System Through HVDS Concepts. Sustainable Energy and Intelligent Systems (SEISCON). 2011: 86-90.

[4] Feng Xiaoke, Shen Weiwei, Dong Chunyu, Zeng Jianliang, Shi Jidong, Wang Dongju. Study on Fundamental Principles and Methodologies of Distribution Network Reconfiguration. TELKOMNIKA Indonesian Journal of ElectricalEngineering. 2014; 12(3): 1695-1700.

[5] K Amaresh, S Sivanagaraju, V Sankar. Minimization of Losses in Radial Distribution System by using HVDS. Power Electronics, Drives and Energy Systems (PEDES). 2006: 1-5.

[6] Md Sarwar, Anwar Shahzad Siddiqui, Zainul A Jaffery, Imran Ahmad Quadri. Techno-Economic Feasibility of HVDS Concept for Distribution Feeder Power Loss Minimisation. Power Electronics (IICPE). India. 2012: 1-4.

[7] P Ravi Babu, Sushma B, Ashwin Kumar B. HVDS approach for reducing the Technical and Nontechnical losses to enhance the Electrical Distribution System performance. Power Electronics (IICPE). India. 2012: 1-5.

[8] K Spandana, Varsha Reddy A. Restructuring of a Low Voltage Distribution System into a High Voltage Distribution System for an Improved Voltage and Power Loss Profile. Green Energy for Sustainable Development (ICUE). 2014: 1-7.

[9] S Naveen, K Sathish Kumar, K Rajalakshmi. Distribution system reconfiguration for loss minimization using modified bacterial foraging optimization algorithm. Electrical Power and Energy Systems. 2015; 69: $90-97$.

[10] A Mohamed Imran, M Kowsalya. A new power system reconfiguration scheme for power loss minimization and voltage profile enhancement using Fireworks Algorithm. Electrical Power and Energy Systems. 2014; 62: 312-322.

[11] GB Dabre, AA Dutta, AN Kadu. Performance Evaluation of Distribution Network and Reduction in Technical \& Non-Technical Losses by Using Energy Efficient Equipment and Cost Benefit Analysis in The Power Sector. Power, Automation and Communication (INPAC). 2014: 6-11.

[12] Dina Khaniya, Anurag K. Srivastava, Noel N Schulz. Distribution Power Flow for Multiphase Meshed or Radial Systems, Power Symposium. NAPS '08. 40th North American. 2008: 1-5.

TELKOMNIKA Vol. 16, No. 3, December 2015: $431-438$ 